\title{
Proper Motions in the Andromeda Subgroup
}

\author{
Andreas Brunthaler ${ }^{1}$, Mark J. Reid ${ }^{2}$, Heino Falcke ${ }^{3,4}$, Christian Henkel ${ }^{1}$ and \\ Karl M. Menten ${ }^{1}$ \\ 1 Max-Planck-Institut für Radioastronomie, Auf dem Hügel 69, 53121 Bonn, \\ Germany \\ 2 Harvard-Smithsonian Center for Astrophysics, 60 Garden Street, Cambridge, \\ MA 02138, USA \\ 3 Department of Astrophysics, Radboud Universiteit Nijmegen, Postbus 9010, \\ 6500 GL Nijmegen, The Netherlands \\ 4 ASTRON, Postbus 2, 7990 AA Dwingeloo, the Netherlands
}

This article presents results of VLBI observations of regions of $\mathrm{H}_{2} \mathrm{O}$ maser activity in the Local Group galaxies M33 and IC 10. Since all position measurements were made relative to extragalactic background sources, the proper motions of the two galaxies could be measured. For M33, this provides this galaxy's three dimensional velocity, showing that this galaxy is moving with a velocity of $190 \pm 59 \mathrm{~km} \mathrm{~s}^{-1}$ relative to the Milky Way. For IC 10, we obtain a motion of $215 \pm 42 \mathrm{~km} \mathrm{~s}^{-1}$ relative to the Milky Way. These measurements promise a new handle on dynamical models for the Local Group and the mass and dark matter halo of Andromeda and the Milky Way.

\section{Introduction}

The problem when trying to derive the gravitational potential of the Local Group is that usually only radial velocities are known and hence statistical approaches have to be used. Kulessa and Lynden-Bell introduced a maximum likelihood method which requires only the line-of-sight velocities, but it is also based on some assumptions (eccentricities, equipartition) (1).

Clearly, the most reliable way of deriving masses is using orbits, which requires the knowledge of three-dimensional velocity vectors obtained from measurements of proper motions. However, measuring proper motions of members of the Local Group is difficult. In recent years, the proper motions of a number of Galactic satellites have been measured using the HST ( 2 and references therein). These galaxies are all closer than $150 \mathrm{kpc}$ and show motions between 0.2 and a few milliarcseconds (mas) per year. More distant galaxies, such as galaxies in the Andromeda subgroup at distances of $\sim 800 \mathrm{kpc}$, have smaller angular motions, which are currently not measurable with optical telescopes. 


\section{Proper Motions of M33 and IC 10}

We observed $\mathrm{H}_{2} \mathrm{O}$ maser emission from two star-forming regions in the disk of M33 associated with the HII region complexes M33/19 and IC 133 eight times with the $\mathrm{NRAO}^{5}$ Very Long Baseline Array (VLBA) between March 2001 and June 2005 (3). We observed the usually brightest maser in IC 10-SE with the VLBA thirteen times between February 2001 and June 2005 (4).

The motions of 4 components in M33/19 and 6 components in IC 133 could be followed over all epochs. The component identification was based on the positions and radial velocities of the maser emission. A rectilinear motion was fit to each maser feature in each velocity channel separately. Then, the variance weighted average of all motions was calculated. This yields an average motion of the maser components in $\mathrm{M} 33 / 19$ of $35.5 \pm 2.7 \mu \mathrm{as} \mathrm{yr}^{-1}$ in right ascension and $-12.5 \pm 6.3 \mu \mathrm{as} \mathrm{yr}^{-1}$ in declination relative to the background source J0137+312. For IC 133 one gets an average motion of $4.7 \pm 3.2 \mu$ as $\mathrm{yr}^{-1}$ in right ascension and $-14.1 \pm 6.4 \mu \mathrm{as} \mathrm{yr}^{-1}$ in declination.

The observed proper motion of a maser region in M33 can be decomposed into the motion of the masers due to the internal galactic rotation in M33, the apparent motion of M33 caused by the rotation of the Sun around the Galactic Center, and the true proper motion of M33 relative to the Galaxy. Since the motion of the Sun and the rotation of M33 (5) are known, one can calculate the true proper motion of M33: $-101 \pm 35 \mathrm{~km} \mathrm{~s}^{-1}$ in right ascension and $156 \pm 47 \mathrm{~km} \mathrm{~s}^{-1}$ in declination, relative to the center of the Milky Way.

Finally, the systemic radial velocity of M33 is $-179 \mathrm{~km} \mathrm{~s}^{-1}$. The radial component of the rotation of the Milky Way toward M33 is $-140 \pm 9 \mathrm{~km} \mathrm{~s}^{-1}$. Hence, M33 is moving with $-39 \pm 9 \mathrm{~km} \mathrm{~s}^{-1}$ toward the Milky Way. Combining the proper motions and radial velocities, gives the three dimensional velocity vector of M33. The total velocity of M33 relative to the Milky Way is $190 \pm$ $59 \mathrm{~km} \mathrm{~s}^{-1}$.

In IC 10 only the strongest maser component was detected in all epochs. Rectilinear motion was fit to the data and yielded a value of $6 \pm 5 \mu \mathrm{as} \mathrm{yr}^{-1}$ toward the East and $23 \pm 5 \mu \mathrm{as} \mathrm{yr}^{-1}$ toward the North. Once again, the contributions of the known motion of the Sun and the known rotation of IC 10 (6) can be calculated. The true proper motion of IC 10 is $-122 \pm 31 \mathrm{~km} \mathrm{~s}^{-1}$ in right ascension and $97 \pm 27 \mathrm{~km} \mathrm{~s}^{-1}$ in declination.

The measured systematic heliocentric velocity of IC $10\left(-344 \pm 3 \mathrm{~km} \mathrm{~s}^{-1}\right)$ is the sum of the radial motion of IC 10 toward the Sun and the component of the solar motion about the Galactic Center toward IC 10 which is $-196 \pm 10$ $\mathrm{km} \mathrm{s}^{-1}$. Hence, IC 10 is moving with $148 \pm 10 \mathrm{~km} \mathrm{~s}^{-1}$ toward the Sun. The proper motion and the radial velocity combined give the three-dimensional space velocity of IC 10 . The total velocity is $215 \pm 42 \mathrm{~km} \mathrm{~s}^{-1}$ relative to the Milky Way.

\footnotetext{
${ }^{5}$ The National Radio Astronomy Observatory is operated by Associated Universities, Inc., under a cooperative agreement with the National Science Foundation.
} 


\section{Local Group Dynamics and Mass of M31}

If IC 10 or M33 are bound to M31, then the velocity of the two galaxies relative to M31 must be smaller than the escape velocity and one can deduce a lower limit on the mass of M31:

$$
M_{M 31}>\frac{v_{r e l}^{2} R}{2 G} .
$$

A relative velocity of $147 \mathrm{~km} \mathrm{~s}^{-1}$ - for a zero tangential motion of M31 and a distance of $262 \mathrm{kpc}$ between IC 10 and M31 gives a lower limit of 6.6 $\times 10^{11} \mathrm{M}_{\odot}$. One can repeat this calculation for any tangential motion of M31. The results are shown in Fig. 1 (top). The lowest value of $0.7 \times 10^{11} \mathrm{M}_{\odot}$ is found for a tangential motion of M31 of $-130 \mathrm{~km} \mathrm{~s}^{-1}$ toward the East and 35 $\mathrm{km} \mathrm{s}^{-1}$ toward the North.

For a relative motion of $230 \mathrm{~km} \mathrm{~s}^{-1}$ between M33 and M31 - again for a zero tangential motion of M31 - and a distance of $202 \mathrm{kpc}$, one gets a lower limit of $1.2 \times 10^{12} \mathrm{M}_{\odot}$ (3). Fig. 1 (top) shows also the lower limit of the mass of M31 for different tangential motions of M31 if M33 is bound to M31. The lowest value is $4 \times 10^{11} \mathrm{M}_{\odot}$ for a tangential motion of M31 of $-115 \mathrm{~km} \mathrm{~s}^{-1}$ toward the East and $160 \mathrm{~km} \mathrm{~s}^{-1}$ toward the North.

In (7) it was found that proper motions of M31 in negative right ascension and positive declination would have lead to close interactions between M31 and M33 in the past. These proper motions of M31 can be ruled out, since the stellar disk of M33 does not show any signs of strong interactions.

Thus, we can rule out certain regions in Fig. 1. This results in a lower limit of $7.5 \times 10^{11} \mathrm{M}_{\odot}$ for M31 and agrees with a recent estimate of $12.3_{-6}^{+18} \times$

$10^{11} \mathrm{M}_{\odot}$ derived from the three-dimensional positions and radial velocities of its satellite galaxies (8).

Acknowledgements: This research was supported by the DFG Priority Programme 1177.

\section{References}

[1] Kulessa A. S., Lynden-Bell D., 1992, MNRAS, 255, 105

[2] Piatek S., Pryor C., Bristow P., et al., 2006, AJ, 131, 1445

[3] Brunthaler A., Reid M. J., Falcke H., Greenhill L. J., Henkel C., 2005, Science, 307, 1440

[4] Brunthaler A., Reid M. J., Falcke H., Henkel C., Menten K. M., 2007, A\&A, 462,101

[5] Corbelli E., Schneider S. E., 1997, ApJ, 479, 244

[6] Wilcots E. M., Miller B. W., 1998, AJ, 116, 2363

[7] Loeb A., Reid M. J., Brunthaler A., Falcke H., 2005, ApJ, 633, 894

[8] Evans N. W., Wilkinson M. I., 2000, MNRAS, 316, 929 

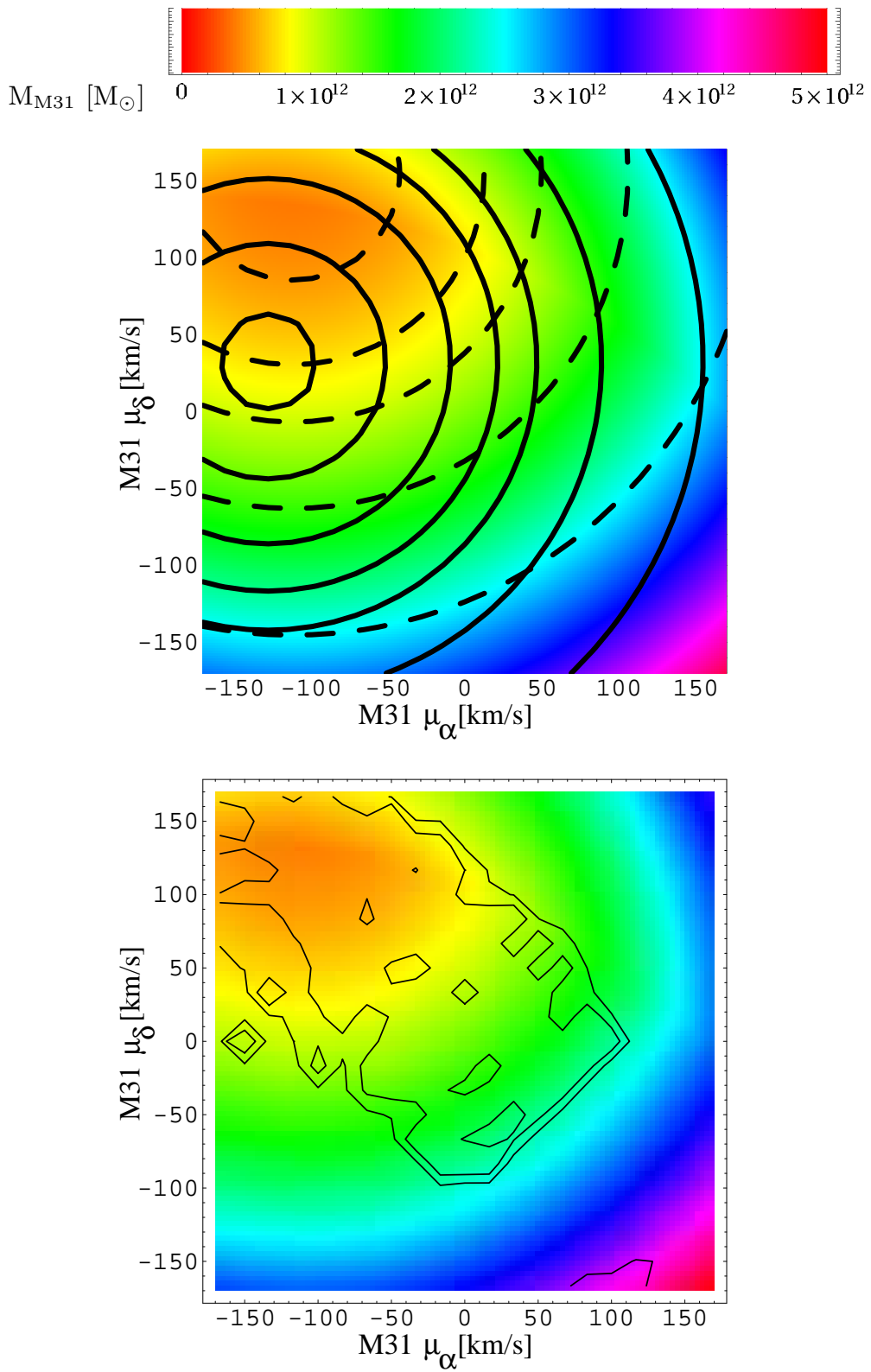

Fig. 1. Top: Lower limit on the mass of M31 for different tangential motions of M31 assuming that M33 (dashed) or IC 10 (solid) are bound to M31. The lower limits to the mass of M31 are $(4,5,7.5,10,15,25) \times 10^{11} \mathrm{M}_{\odot}$ for M33, and $(0.7,1,2.5,5$, $7.5,10,15,25) \times 10^{11} \mathrm{M}_{\odot}$ for IC 10 , rising from inside. The colour scale indicates the maximum of both values. Bottom: The colour scale is the same as above and gives the lower limit on the mass of M31. The contours show ranges of proper motions that would have led to a large amount of stars stripped from the disk of M33 through interactions with M31 or the Milky Way in the past. The contours delineate 20\% and $50 \%$ of the total number of stars stripped (7). These regions can be excluded, since the stellar disk of M33 shows no signs of such interactions. Taken from (4). 